variance in confidence and knowledge about working with people with a learning disability at end-of-life.

- Shared teaching programme commenced.

- New resources available for hospice staff, and patients.

- Engagement within the hospice environment - inpatient and community.

- Engagement with community services, experts by experience, care providers, community groups to plan events and start the conversation about death and dying.

- Increased recognition of the needs of relatives with a learning disability when caring for a parent, sibling, co-resident etc.

\section{P-12 COLLABORATIVE WORKING WITH LEARNING DISABILITIES PATIENT EXPERIENCE GROUP}

Joanne Schofield. The Prince of Wales Hospice, Pontefract, UK

10.1136/spcare-2021-Hospice.33

In 2019 the hospice was contacted to provide easy-read literature explaining what hospice care is for a person with a learning disability. As an organisation we didn't have this kind of documentation and we couldn't source it from elsewhere. This set us on a journey of collaborative working and service co-design with our local Community Learning Disabilities Team and Learning Disabilities Patient Experience Group (LDPEG).

The first draft of the leaflet was utterly 'trashed' by the group; they were particularly critical of the images we had used as none of them were of our hospice building and none of them contained people with a learning disability. As a result, a number of the LDPEG visited the hospice and met with staff. They proved very insightful and challenging in their questioning of the work we do as well as about death and dying. We were in the process of finalising the leaflet, using members of the LDPEG in the photographs as well as piloting a wellbeing group when COVID-19 hit and everything had to be postponed.

We are now in a position to re-commence that work. We have an afternoon tea event scheduled for 23 June to celebrate Learning Disability Week and will hopefully be in a position to complete the easyread literature before Hospice UK's conference in November. In talking to professionals and carers we are aware how challenging advance care planning discussions can be, and we hope to pilot a group for people with learning disabilities and their families/carers to introduce hospice care and advance care planning discussions. This will hopefully address some of the shocking inequalities in end-of-life care people with a learning disability can experience.

We also plan to provide bereavement support, not only to families and informal carers. but also to paid carers who may have had a longstanding relationship with an individual prior to their death.

\section{P-13 THE LEARNING DISABILITY DEATH REVIEW (LEDER) PROGRAMME IN A UK HOSPICE}

Nick Dando. Phyllis Tuckwell Hospice Care, Farnham, UK
Background The Learning Disability Death Review (LeDeR) was commissioned by NHS England in 2015 in response to the 2013 Confidential Inquiry into the Premature Deaths of people with Learning Disability (CIPOLD) (Heslop, Blair, Fleming, et al., 2014). Phyllis Tuckwell Hospice Care (PTHC) integrated the LeDeR programme into our learning from deaths process in 2019.

Aims This report shares our experience of undertaking reviews of the care provided to patients with a learning disability and outlines the enhanced working relationships which have resulted from our engagement with the LeDer programme.

Methods PTHC reported our first patient death to the LeDeR programme in October 2019 and conducted a Structured Judgement Review (SJR) in line with NHS (NHS Improvement, 2018) and Royal College of Physicians (2016) guidelines. We have subsequently reviewed the deaths of seven other patients with a learning disability between April 2019 and March 2021.

Results Of the eight cases reviewed, five patients were cared for at the end-of-life in their usual place of residence - considered a marker of good practice - with three supported on the inpatient unit. Other examples of good practice include documented mental capacity assessment and best-interests decision-making on admission or first community review and evidence of reasonable adjustments including adaptation to room lighting and supporting a pet to visit.

One case identified important areas for improvement with limited evidence of mental capacity assessment and delayed access to appropriately funded care. The case was reviewed at a multi-professional meeting with action points disseminated within the hospice and shared with the regional LeDeR team. Conclusions Training on mental capacity assessment in learning disabilities has been included in educational meetings and incorporated into organisational mandatory training. PTHC is now a core member of the regional LeDeR team and has provided training in end-of-life care to the learning disabilities team in our local Community Mental Health Trust. These links help us reach and enhance the care for more patients with learning disabilities.

\section{P-14 THE CASCADE PROJECT: PROMOTING AGE-ATTUNED PALLIATIVE CARE}

${ }^{1}$ Helen King, ${ }^{1}$ Heather Richardson, ${ }^{2}$ Caroline Nicholson. ${ }^{1}$ St Christopher's Hospice, London, UK; ${ }^{2}$ University of Surrey, Guildford, UK

\subsection{6/spcare-2021-Hospice.35}

Background It is now widely recognised that the palliative care needs of older people with frailty are poorly met compared to those of others at the end-of life (Hamaker, van den Bos, Rostoft, 2020). More people die of frailty and age related comorbidities than of cancer or heart disease and yet they are not the focus of end-of-life care. The COVID-19 pandemic has emphasised the difficulties in providing the right care at the right time for this particular disadvantaged group (Lebrasseur, Fortin-Bédard, Lettre, Raymond, et al., 2021).

Aim To improve confidence and capability in (a) hospice and (b) community sectors in understanding and delivering ageattuned palliative care.

Method We implemented the approach described in Ageattuned Hospice Care (Nicholson \& Richardson, 2018). Three 
phases: (1) Focus groups with key stakeholders, July- Nov 2019; (2) Establish special interest groups to develop resources, July 2020 onwards; (3) Disseminate information locally and nationally, May 2021 onwards.

Results The focus groups identified three core capabilities: (1) Assessment of frailty and palliative care needs, (2) Recognition of deterioration and symptom management, (3) Communication around advance care planning (ACP). We established six special interest groups related to frailty: recognition; resource mapping; partnerships; rehabilitation in care homes; dementia; and ACP.

Activities to date include: implementing frailty identification as routine practice across the hospice including adaption of electronic systems to plan and audit care, creating proformas for multidisciplinary meetings with GPs and DNs focusing on frailty; devising crib cards for ACP conversations using $\mathrm{CLaD}$ intervention methodology and management of frailty related symptoms e.g. delirium and building capability with local partners in rehabilitation and recognition of palliative care need. Early evaluation suggests wide spread adoption and increased confidence and understanding of frailty.

Conclusion We have shown that the palliative care needs of older people with frailty can be enhanced by highlighting information and resources. We plan to cascade further through local and national conferences.

\section{P-15 THE ROLE OF PROJECT ECHO IN BUILDING COMMUNITIES OF PRACTICE TO SUPPORT MENTAL HEALTH AND WELLBEING}

${ }^{1}$ Ruth Gray, ${ }^{2}$ Chris Jenkins, ${ }^{3}$ Helen McNally, ${ }^{2}$ Tracey McTernaghan. ${ }^{1}$ SE Health and Social Care Trust, Belfast, UK; ${ }^{2}$ Hospice UK, Belfast, UK; ${ }^{3}$ Ulster University, Newtownabbey, UK

10.1136/spcare-2021-Hospice.36

Background People living in prison may experience poor mental health, psychological disorders, and frequently have experienced traumatic episodes as both children and adults. As a post-conflict setting with high levels of Post-traumatic stress disorder in the general population, these challenges are likely particularly evident within Northern Ireland's prison population.

Aim(s) The Northern Ireland Prison Service within the Department of Justice is responsible for delivering prison services, while the South Eastern Health and Social Care Trust is responsible for delivering health care in the region's prisons. This study sought to assess whether Project ECHO is a useful tool for building communities of practice across agencies to address challenges in supporting mental health and wellbeing services within Northern Ireland's prisons.

Methods Project ECHO (Extension for Community Healthcare Outcomes) is a tele-mentoring programme that uses video-conferencing technology to deliver evidence-based, best practice guidance and education; and case-based learning through presentations by network participants. Interviews were conducted with members of the Northern Ireland Prisons ECHO network on the impact of the programme, and analysed thematically.

Results The ECHO approach was considered effective by participants in bridging knowledge gaps between different levels, service providers, and institutions involved in delivering mental health services in prisons. Wider changes in service delivery, catalysed through the relationships developed within ECHO, were described as encouraging a greater focus on client-centred planning. The ECHO network helped to catalyse other important changes within the prison service such as the development of a new referral pathway between the Northern Ireland Prison Service and the South Eastern Health and Social Care mental health team, and new operating procedures for keeping people safe in custody.

Conclusions This study show the value of digital-based solutions such as Project ECHO to build communities of practice between different institutions to achieve goals such as improving mental health and wellbeing in a complex prison population.

\section{P-16 SOCIAL PRESCRIBING AT END OF LIFE WITH A FOCUS ON THOSE WHO DO NOT TRADITIONALLY ACCESS HOSPICE CARE}

${ }^{1}$ Nicola Button, ${ }^{1}$ Maureen Hanley, ${ }^{2}$ Louise Willsher, ${ }^{3}$ Lisa Andrews, ${ }^{1}$ Lisa Parrish. ${ }^{1}$ St Helena Hospice, Colchester, UK; ${ }^{2}$ C360, Colchester, UK; ${ }^{3}$ CVST, Clacton, UK

\subsection{6/spcare-2021-Hospice.37}

In collaboration with both local Community Voluntary Services (CVS), Community 360, Community Voluntary Services Tendring and Essex Faith Covenant, St Helena Hospice has been successful in receiving grant funding from the Masonic Charitable Foundation for an exciting new project. This has further been supported financially by our Alliance End of Life Board.

In this Social Prescribing ${ }^{\mathrm{TM}}$ pilot project, we will recruit two additional engagement officers, one in both Colchester and Tendring to work with under-represented Black, Asian and minority ethnic and deprived groups, and any other communities who do not traditionally access hospice care. We will work to better understand their needs and barriers to accessing services. The Engagement Officers will be given training on social prescribing and end-of-life care and will work across CVSs and the hospice.

Our dashboard shows inequity of access for those living in areas of high deprivation and this project will link with our well established SafeHarbour project.

The Engagement Officers will work with existing services in the community to develop an awareness of the needs of these communities and propose modifications which would promote greater equality of access as well as supporting these communities to feel better informed at supporting their citizens at end-of-life. If gaps are identified within local community assets we will work with partners to develop, support and where appropriate seek investment in community assets. This collaborative approach would enable communities to co-produce solutions to improve health outcomes, with the Engagement Officer serving as a facilitator across community networks.

We hope community leaders will gain a better knowledge of what happens at end-of-life and feel empowered to shape the services available. Equally end-of-life providers would learn more about the community services available to support their patients and how our services need to be adapted in order to ensure equality of access for all. 